\title{
BMJ Open Implementation of music in the perioperative standard care of colorectal surgery: study protocol of the IMPROVE Study
}

\author{
Ellaha Kakar (D) ,1,2 Erwin Ista, ${ }^{2,3,4}$ M Klimek (D) ,5 Johannes Jeekel ${ }^{1,6}$
}

To cite: Kakar E, Ista $\mathrm{E}$, Klimek M, et al. Implementation of music in the perioperative standard care of colorectal surgery: study protocol of the IMPROVE Study. BMJ Open 2021;11:e051878. doi:10.1136/ bmjopen-2021-051878

- Prepublication history and additional supplemental material for this paper are available online. To view these files, please visit the journal online (http://dx.doi.org/10.1136/ bmjopen-2021-051878).

Received 31 March 2021 Accepted 15 October 202

Check for updates

(c) Author(s) (or their employer(s)) 2021. Re-use permitted under CC BY-NC. No commercial re-use. See rights and permissions. Published by BMJ.

${ }^{1}$ General Surgery and Intensive Care Unit, Erasmus MC Rotterdam, Zuid-Holland, The Netherlands

${ }^{2}$ Intensive Care Unit, Erasmus MC, Rotterdam, Netherlands ${ }^{3}$ Pediatric surgery, Intensive Care Unit, Internal Medicine, Erasmus MC, Rotterdam, ZuidHolland, The Netherlands

${ }^{4}$ Internal Medicine, Erasmus MC, Rotterdam, Netherlands

${ }^{5}$ Anesthesiology, Erasmus MC, University Medical Centre,

Rotterdam, The Netherlands

${ }^{6}$ Neuroscience, Erasmus MC, Rotterdam, Netherlands

Correspondence to

Ellaha Kakar;

e.kakar@erasmusmc.nl

\section{ABSTRACT}

Introduction Perioperative music intervention has been proven effective in reducing anxiety, pain, neurohormonal stress response and medication requirement.

Unfortunately, there is a gap between new effective interventions and their (interventions) integration in standard care protocols. The aim of this preimplementation and postimplementation study is to investigate the adherence to a music intervention in a tailored, multilevel, systematic implementation strategy and the initial impact of the implementation on postoperative pain in patients undergoing colorectal surgery.

Methods and design A monocentre prospective preimplementation and postimplementation study was set up using The Consolidated Framework for Implementation Research to conduct a systematic implementation of music intervention in three phases. Primary outcomes are adherence to the music intervention and the initial impact of the music intervention implementation on postoperative pain scores on the first postoperative day. At least 100 patients will be included in the study. Secondary outcomes include adherence to the implementation strategy, penetration of music intervention in the standard care, assessment of the determinants for implementation, impact of the implemented music intervention on pain during the entire admission, anxiety, medication usage, complications, hospital and intensive care unit (ICU) length of stay.

Ethics and dissemination The Medical Ethics

Review Board of Erasmus MC University Medical

Centre Rotterdam, The Netherlands, has approved this protocol. The study is being conducted in accordance with the Declaration of Helsinki. Results of this trial will be published in peer-reviewed scientific journals and conference presentations.

Trial registration Dutch Trial Register NL8071.

\section{INTRODUCTION}

Patients undergoing surgical procedures often experience perioperative anxiety, pain and stress which may lead to complications, delayed postoperative recovery and extended hospitalisation. $^{1-10}$ Furthermore, it is also known that preoperative anxiety leads to higher postoperative pain levels, further increasing the risk of the unwanted outcomes
Strength and limitations of this study

- This study protocol is the first implementation study of perioperative music intervention in a surgical population and can serve as a guideline for future implementation projects for music interventions.

- Implementation strategies are based on the assessment of barriers for implementation on multiple levels and in which, during the process evaluation, the strategy can be adapted in order to achieve the highest possible efficacy.

- The study is powered to assess the impact of implementing music intervention on an important perioperative health outcome (postoperative pain), although due to the design of the study (preimplementation and postimplementation study) the impact may not be detected.

- Patients and professionals cannot be blinded for the intervention because it may lead to performance and detection bias.

mentioned before. ${ }^{11}$ Patients with inflammatory bowel disease (IBD) and cancer are known to have higher anxiety rates than the general population. ${ }^{12-14}$ Many of these patients undergo major surgical procedures, which cause additional psychological stressors. ${ }^{15}{ }^{16}$ Recent systematic reviews of randomised controlled trials have shown significant positive effects on listening of recorded music interventions on preoperative anxiety, postoperative pain, intraoperative sedative and postoperative analgesic medication requirement, as well as postoperative neurohormonal stress reaction. ${ }^{17-19}$

Pharmacological agents, such as benzodiazepines and opioids, are widely used as treatment for anxiety and pain, even though the side effects and risks are well known..$^{20-24}$ Preoperative benzodiazepines to reduce anxiety have been associated with worse postoperative recovery and higher risk for delirium. ${ }^{625} 26$ Also, the use of benzodiazepines and opioids in the hospital may lead to 
chronic use, which is partly accountable for the emerging opioid epidemic. ${ }^{27}$ Music as a non-pharmaceutical intervention on the other hand is relatively inexpensive, easily applicable and without any side effects known. Furthermore, given the evidence for potential reduction in previously mentioned factors, music should be considered as part of the perioperative standard care procedure in hospitals

Studies aiming to implement music intervention in the standard care procedure until now only reached an adherence rate of $36 \%-53 \%,{ }^{29-31}$ which is considered low for significant effects of the intervention. This is caused by several barriers for implementation which were not overcome before implementation, which is essential for an effective implementation.

Thus, the Implementation of Music intervention in the PeRiOperatiVe standard carE (IMPROVE) Study will be set up to achieve tailored implementation of music intervention in perioperative standard care in a systematic manner.

\section{AIMS AND OBJECTIVES}

The primary aim of the IMPROVE Study is to assess the adherence to the music intervention (if the intervention is provided as advised) ${ }^{32}$ and the initial impact of the music intervention on the first postoperative day (POD) pain scores. Secondary aims are the penetration of the intervention, adherence to the tailored implementation strategy (if eg, information to the patient on time during the outpatient clinic visit, as part of the tailored strategy, is provided as developed), facilitators and barriers for implementation, impact of the implemented music intervention on postoperative pain during the entire hospitalisation, anxiety, perioperative pain medication usage, intraoperative analgesic and sedative medication usage, complications, and hospital and intensive care unit length of stay (LOS).

\section{METHODS AND ANALYSIS}

The IMPROVE Study is a prospective, monocentre, study carried out in the IJsselland hospital, Capelle aan den IJssel, the Netherlands. Implementation will be carried out using The Consolidated Framework for Implementation Research (CFIR), ${ }^{33}$ which provides a construct associated with effective implementation and is a commonly used framework in the field of implementation. ${ }^{34}$ The CFIR assesses five domains; (1) Intervention characteristics, (2) Outer setting, (3) Inner setting, (4) Characteristics of the individuals involved, and (5) The process of implementation. Based on this the study encompasses three phases. ${ }^{33}$

\section{Study phases}

Phase I (domains 1, 2, 3 and 4)

Evaluation of the characteristics of the intervention, current practice regarding anxiety and pain management, in-hospital music application, and identification of facilitators and barriers for implementation of recorded music in the elective colorectal standard of care procedure.

\section{Phase II}

Based on the results of phase I we will develop a tailored implementation strategy for the context (recorded music intervention) and setting (elective perioperative standard care procedure of colorectal surgery in the IJsselland Hospital).

\section{Phase III (domain 5)}

Finally, the tailored implementation strategy will be applied and the effect of the intervention on implementation outcomes, for example, adherence to the music intervention during perioperative care, and clinical outcomes will be assessed.

In the next section each phase is described in detail, including the setting, study design, procedures, outcome measures and statistical analysis. Figure 1 gives an overview of the study phases.

\section{Setting}

The gastrointestinal (GI) surgery ward was chosen as a first site of implementation in the Netherlands due the relative vulnerable patient population, as patients with IBD and colorectal cancer experience higher levels of anxiety and the standard care procedure is more standardised due to the Enhanced Recovery After Surgery protocol. ${ }^{35}$ Patients are eligible when aged $\geq 18$ years, undergoing colorectal surgical procedure for malignant or benign (mostly IBD) disease, and admitted to the GI nursing ward (excluding day care ambulatory interventions).

A dedicated team of healthcare professionals of the implementation site involved in the perioperative process will be assembled (engagement). The dedicated team will exist out of one person from each group of professionals; GI surgeon, GI surgery ward nurse, anaesthesiologist, anaesthesiology assistant, specialised nurse in GI oncology and a specialised nurse in Stoma Care. The dedicated team will actively engage in the study in order to reach effective implementation of the intervention in daily practice. The dedicated team will be assisted by the research team, which includes an implementation expert.

\section{Phase I}

Evaluation of the current practice regarding pain and anxiety management, in-hospital music application, and identification of facilitators and barriers for implementation of recorded music in the elective colorectal standard of care procedure.

\section{Study design}

We will assess prospectively the current practice regarding anxiety and pain management in healthcare professionals involved in the perioperative standard care using anonymous electronic surveys. Identification of barriers and facilitators will be also assessed prospectively in patients and healthcare professionals using CFIR. These results 


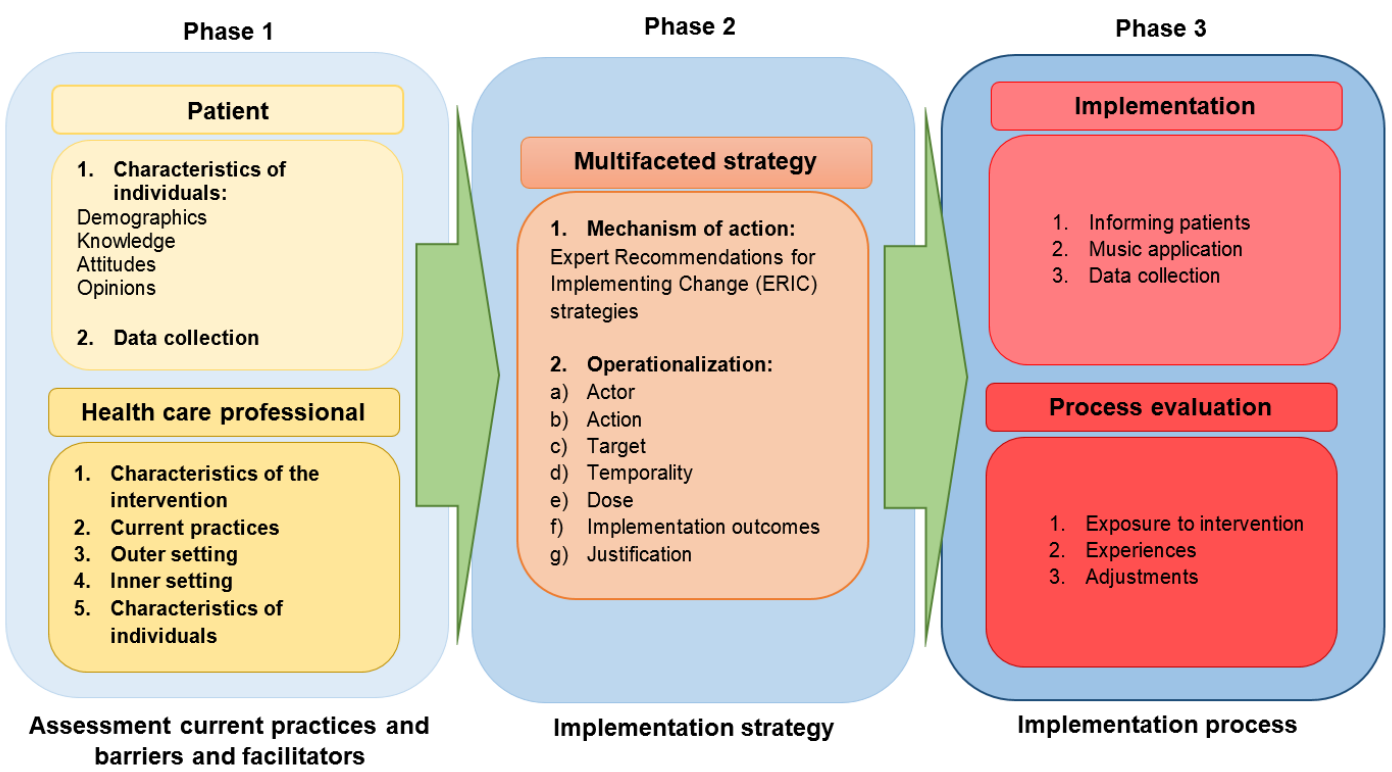

Figure 1 Overview of the study phases.

will serve as baseline measures to compare with the outcomes of the implementation process.

\section{Measures}

Current practice regarding anxiety and pain management in the colorectal surgery population and assessment of facilitations and barriers for implementing perioperative music intervention in the standard of care will be evaluated by using qualitative research methods in the form of surveys in patients and healthcare professionals involved in the perioperative standard of care procedure.

\section{Study population}

All patients who have undergone a colorectal surgical procedure for benign (mostly IBD) disease or cancer will be informed regarding the study and surveyed, after written informed consent is acquired for data collection from the patients' medical file, on the first POD. Patients will be screened by the admission agency of the surgery department. Eligible patients will be asked by the attending physician if the research team can approach them regarding the IMPROVE Study. At last, a member of the research team will inform and acquire informed consent. As mentioned earlier, healthcare professionals involved in the perioperative process, including general surgeons, anaesthesiologists, GI surgery ward and anaesthesiology nurses, specialised nurses in GI oncology and Stoma Care, and surgery and anaesthesiology residents, will also be surveyed in this phase. The electronic survey was sent via email to members of the dedicated team. The survey will be then further distributed among all professionals by the dedicated team. Reminders will be sent via email every month.

\section{Surveys}

An online self-made survey will be conducted in patients and healthcare professionals. The complete surveys for this phase can be found in online supplemental file 1 (patient survey) and online supplemental file 2 (healthcare professional survey). The surveys are based on four domains of CFIR; inner setting, outer setting, and characteristics of the individuals and intervention. The outer setting includes assessment of patient needs and peer pressure (need for hospitals to have the same policies). The inner setting mostly includes assessment of characteristics of the hospital/implementation site, including network and communication, culture (norms and values), knowledge, current use of the intervention, and implementation climate (tension for change, feedback, learning climate, etc). Patients will be surveyed on their preferences, beliefs and opinions regarding recorded music in the perioperative healthcare and will be asked to score their anxiety levels in the preoperative and postoperative period. Healthcare professionals will be surveyed on the current practice and beliefs regarding anxiety and pain management and characteristics of the site; for example, communication, schooling, leadership and so on, and characteristics of the intervention, for example, easy to implement and so on. Furthermore, the surveys will contain questions regarding knowledge on the current information regarding the effect of music in medical healthcare and demographic characteristics of the responders. The patient survey will mostly assess patient needs (outer setting) and characteristics of the individuals. The healthcare professional survey will assess aspects of the outer and inner settings and characteristics of the intervention and individuals involved.

\section{Survey validity}

To ensure face and content validity of the healthcare professional survey, the first drafts were carefully revised by members of the research team (EK/EI). After consensus was reached, the healthcare professional survey was 
presented to a dedicated team at the study site, consisting of a surgeon, anaesthesiologist, nurse and nurse practitioner for input. Two colleagues with a medical background assessed the survey as well. Any comments were incorporated in a new version.

\section{Data analysis}

Response rate will be defined as the percentage of all approached professionals who finish the second category of the survey. Respondents who do not finish this category (music knowledge) will be excluded from analysis. Descriptive statistics will be used to describe the outcomes of the current practices regarding pain, anxiety and sleep managements. Continuous data will be presented using medians and IQRs. The Kruskal-Wallis test will be used to analyse differences between groups (healthcare professionals, age and working experience). Categorical data will be analysed using $\chi^{2}$ tests if group samples are at least five, and Fischer's exact tests if at least one group sample is less than five. Ordinal data (eg, age and working experience) will be analysed using the Kruskal-Wallis test. The Bonferroni correction will be applied in case of multiple testing in the post hoc tests. Self-conducted Median Knowledge Scores (MKS) will be calculated for the patients and professionals; these will be presented as medians (IQR).

The definitions of barriers and facilitators will be based on expert opinion (research team). As mentioned earlier, barriers and facilitators will be classified according to the domains of CFIR and expressed in percentages. An MKS under $70.0 \%$ is defined as a barrier. An MKS of $70.0 \%$ or higher will be considered as a facilitator. Other barriers will be defined as less than $50 \%$ of the answers being beneficial to implementation. When this percentage equals or exceeds $50 \%$, this aspect will be treated as a facilitator. Data will be analysed using R studio V.4.0.0.

\section{Phase II: Development of the tailored implementation strategy}

Based on the barriers and facilitators assessed in phase $\mathrm{I}$, two members of the research team $(\mathrm{EK} / \mathrm{EI})$ will select the implementation strategies. For example, if we assess during phase I that the knowledge regarding music intervention in the healthcare is low, this may will be considered as a barrier for implementation. The implementation strategy for this would be developing and distributing educational materials, for example, pocket card for nurses with information about how to apply the intervention, in order to handle this barrier based on the Expert Recommendations for Implementing Change strategies. ${ }^{36}$ The mechanism of action in this case would be to develop and format manuals, toolkits and other supporting materials in ways that make it easier for stakeholders to learn about the innovation and for clinicians to learn how to deliver the clinical innovation and distribute them in person, by mail and/or electronically. ${ }^{36}$ Through discussions with the coauthors and dedicated team these will be fine-tuned in order to reach consensus. The goal is to answer the following two important questions: (1)
Can the barriers and facilitators found be successfully translated into a tailored implementation strategy? and (2) Is the tailored implementation strategy applicable in daily practice? The implementation strategies and mechanisms of actions will then be validated by EI, a coauthor who is an expert on implementation. When consensus among the dedicated team and research team is reached the implementation strategies will be operationalised by using the seven dimensions proposed by Proctor et al..$^{37}$ These dimensions include: the actors(s) - that is, who delivers the strategy?; the action(s); the target(s) of the action-that is, towards what or whom and at what level?; temporality-that is, when or at what phase?; dose-that is, at what frequency and intensity?; the implementation outcome(s) affected; and justification-that is, based on what theoretical, empirical or pragmatic justification? ${ }^{38}$

\section{Phase III: Implementation}

Study design

In the last phase of this implementation study, the music intervention will be put into practice in a single-centre prospective study. The primary aim is to assess the extent to which healthcare professionals can adhere (\%) to the music intervention in elective surgical procedures. Secondary aims are the penetration $(\%)$ of the intervention, adherence $(\%)$ to the tailored implementation strategy, the initial impact (since this is the first implementation study of music in a surgical population) of the music intervention on the first POD pain scores, impact of the implemented music intervention on postoperative pain during the entire hospitalisation, anxiety, perioperative pain medication usage, intraoperative analgesic and sedative medication usage, complications, and hospital and ICU LOS.

\section{Measures}

Adherence to the music intervention will be calculated. Successful implementation is defined as an adherence of $70 \%$ to the music intervention. ${ }^{32} 3940$ The survey used in phase I will be adapted to the postimplementation phase and repeated in order to assess changes in knowledge, opinions and attitudes of the healthcare professionals towards the music intervention as part of the impact of the implementation strategy. Patients who received the music intervention during the implementation phase will be surveyed in order to assess satisfaction regarding the practical use of the music intervention based on the custom-designed implementation strategy and the music intervention itself. Also, the penetration of the intervention in the standard care, defined as the percentage of patients that used the music intervention as advised, will be assessed.

\section{Study population}

Patients who are planned for a surgical procedure for IBD or colorectal cancer will be informed regarding the study preoperatively and receive music intervention perioperatively if they are willing. Exclusion criteria 


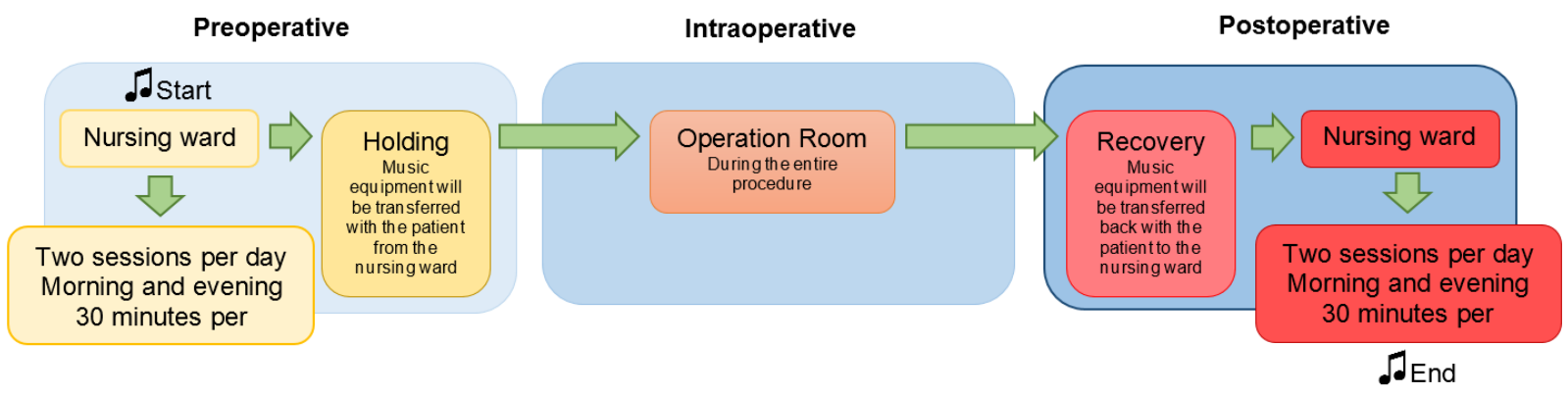

Figure 2 Planned intervention implementation.

will be severe hearing impairment causing problems with verbal communication, patients who are unable or unwilling to receive the intervention, and patients who do not adequately control the Dutch or English language. As mentioned earlier, healthcare professionals involved in the perioperative process, including general surgeons, anaesthesiologists, GI surgery ward and anaesthesiology nurses, specialised nurses in GI oncology and Stoma Care, and surgery and anaesthesiology residents, will also be part of the implementation process.

\section{Procedure}

\section{Music intervention}

Fu et alfound that exposure to at least 120 min of perioperative music, delivered either before, during and/or on the first 2 days after surgery would lead to less opioid requirement. ${ }^{17} \mathrm{~A}$ study in the critically ill population found that music interventions of at least 20-30 min significantly reduced pain scores compared with $10-15$ min. ${ }^{41}$ Based on this our goal is to provide music intervention sessions of at least $30 \mathrm{~min}$, twice a day, during the entire hospital stay in order to achieve clinically relevant effects. Figure 2 shows the planned intervention implementation.

\section{Implementation process}

Eligible patients will be informed preoperatively regarding the use of the music intervention in the perioperative period. The moment of verbally informing the patient will be assessed in the first phase of this study, in which patients' preferences will be assessed in the survey. Multiple methods will be used to inform the patients, for example, folders, information added to existing folders and applications, and so on. Data on adherence will be collected by registration using self-made checklists. The checklist will based on the preceding moments on which the music intervention should be offered and applied by the healthcare professional. Furthermore, patients willing to receive the music intervention will receive the music intervention as planned (figure 2). These patients will then be informed and asked for written informed consent for being surveyed regarding the music intervention they received and data collection on secondary outcome measures from the patients' medical file as described in phase I. This implementation process, in which patients will be provided music during their hospital admission, will go on during a period of 6 months.

\section{Sample size}

Pain management for colorectal surgery in the IJsselland Hospital encompasses patient controlled analgesia (PCA) for laparoscopic surgery and epidural analgesia for primary laparotomic procedures or procedures with a high conversion chance. A minimum reduction of $12 \mathrm{~mm}$ on VAS for pain has been shown as clinically relevant. ${ }^{42}$ To measure the impact of music intervention postimplementation on postoperative pain we performed a sample size calculation based on reported mean pain scores and SD after colorectal surgery in the paper by Kaminski $e t$ $a l$ and Mouawad et at $t^{44}$ considering that pain scores are equivalent to our patient population since scores are based on a large sample and that the surgical procedure and pain management are similar. We used the mean pain scores measured with VAS of patients on the first POD using PCA $(n=173, V A S=4.6 \pm 2.0)$ which yielded the largest sample size. We aim to obtain a power of $80 \%$, with level of significance set at $5 \%(\mathrm{p}=0.05)$, planned two-sided testing and a dropout rate of $10 \%$. Consequently, the sample size will be 50 patients preimplementation and more than 50 patients postimplementation. The implementation process will take 6 months, therefore in the postimplementation period $>50$ patients may be included in the study. Data of all the patients who received the intervention will be used for data analysis after informed consent is acquired.

\section{Evaluation}

During and at the end of the implementation phase the strategy will be reflected and evaluated. Stakeholders in the dedicated team should actively participate in evaluating and improving the implementation strategy as an internal source of the intervention alongside the research team. Monthly a member of the research team will evaluate the adherence to the intervention during the implementation phase. Personal, and if practically possible, team debriefing (focus group) concerning progress and experience of the implementation strategy will be carried out with the healthcare professionals. Based on the measures proposed by Hulscher et at $t^{45}$ process evaluation will be 
carried out. The goal is to assess whether the intended changes are being achieved (eg, exposure to intervention by observation) and the experiences of those exposed (patients and hospital staff). Based on these process evaluations the implementation strategy will be adjusted if found necessary.

\section{Data analysis}

Data will be presented using descriptive statistics. The adherence will be presented as the percentage (\%) of patients who received the music intervention as advised (figure 2). Data on preimplementation and postimplementation, phases 1 and 3, respectively, will be used to assess the initial impact of music intervention in a systematic implementation study on the first POD. The Visual analogue scale (VAS) for pain will be analysed using Student's t-test in case the data are normally distributed, otherwise the Mann-Whitney U test will be used. Data of patients who used the intervention as planned will be used to assess this initial impact. The significance level of all tests will be set at $<0.05$. Differences in the secondary outcomes between preimplementation and postimplementation groups will be analysed using parametrical tests (Student's t-test and $\chi^{2} /$ Fisher's exact tests) for normally distributed data and non-parametrical tests (Mann-Whitney U test and $\chi^{2} /$ Fisher's exact tests).

\section{Patient and public involvement}

Patients or the public were not involved in the design, or conduct, or reporting, or dissemination plans of our research.

\section{ETHICS AND DISSEMINATION \\ Ethical approval}

This study protocol was presented and approved by the Medical Ethical Committee of the Erasmus University Medical Centre (registration number: MEC2019-0563). This study is registered in the "Dutch Trial Register (www.trialregister.nl) under number: NL8071. Data collection will be assessed according to the Dutch Personal Data Protection Regulation. Data will be handled confidentially and anonymously in password protected files and accessed by a limited number of personnel in order to prevent tracing to individual patients.

\section{Informed consent}

All eligible patients in phases I and III of the study will be asked to sign a written informed consent. Informed consent will also encompass participation to the surveys and data extraction on secondary outcome measures from the patients' electronic database, and consent for publication. Data on secondary outcome measures will be used to make comparisons preimplementation and postimplementation of the music intervention.

\section{Dissemination}

Research data can be presented or publicised in agreement with the principal investigator and project leaders only. Research data that can be traced to the individual will not be presented or published. The primary publication will be made by the principal investigator and research team. There are no competing interests between authors. The order of the different authors is not yet known. The funder will have no role in the data collection process, data analysis and interpretation of the trial results.

\section{DISCUSSION}

Music intervention has shown to be effective in various medical fields for several decades now. Unfortunately, it is still not part of the standard medical care, considering it can have positive effects on patient outcomes and is a relatively inexpensive intervention without any known risks. With the current higher level of evidence as mentioned in the introduction there is more reason to implement music in the standard medical care. Therefore, the goal of this study is to implement perioperative music intervention in the standard care process of patients undergoing elective colorectal surgery. First, we will assess the barriers and facilitators for implementing the intervention. Second, based on these barriers and facilitators we will set up a tailored implementation strategy for implementing perioperative music in a specific context and setting.

To the best of our knowledge this is the first implementation study of perioperative music intervention in a surgical population. In this study we used the CFIR implementation framework using rigorous implementation approaches to evaluate the effectiveness of specific methods for implementing. Implementation strategies are based on the assessment of barriers for implementation on multiple levels specific for the setting in which an intervention is implemented. Tailored multilevel strategies are more likely to be effective than single strategies. ${ }^{38}$ Thus, the study is set up in such a way that during the process evaluation the strategy can be adapted, since it is known that flexible and adaptable interventions are more likely to be implemented properly, ${ }^{33}$ in order to achieve high efficacy of the music intervention. Based on this local project we will explore how feasible our implementation strategy is in other sites and settings. The strategy can be adapted during the process evaluation for other sites. Effectivity of the implementation strategies should be studied in a cluster randomised stepped-wedge trial in the future.

Also, the initial impact of implementing music intervention on postoperative pain is assessed, which is an indirect measure of successful implementation. We believe that besides pain, anxiety is also an important outcome parameter. We chose to power the study on pain, since pain has a direct influence on the medication requirement and anxiety may have an indirect influence on medication requirement by its influence on pain. ${ }^{7}$ 
Despite the above, our study also has some limitations. The design of the study (preimplementation and postimplementation study) may be a limitation in finding the effect on clinical outcomes (pain, medication use, etc). These effects may exist but will possibly be not detected due to the design of the study.

In conclusion, this study protocol provides a framework on how to systematically implement perioperative music intervention in the standard care and test the effect of this systematic implementation on the clinically relevant outcome, pain, in surgical patients.

Contributors JJ, El, MK and EK conceived the study design, developed the study procedures and protocol, and drafted the manuscript. All authors have read, edited and approved the final manuscript.

Funding This research was partly funded by the Music as Medicine foundation, Erasmus MC, Rotterdam, The Netherlands, grant number: N/A.

Competing interests None declared.

Patient consent for publication Not applicable.

Provenance and peer review Not commissioned; externally peer reviewed.

Supplemental material This content has been supplied by the author(s). It has not been vetted by BMJ Publishing Group Limited (BMJ) and may not have been peer-reviewed. Any opinions or recommendations discussed are solely those of the author(s) and are not endorsed by BMJ. BMJ disclaims all liability and responsibility arising from any reliance placed on the content. Where the content includes any translated material, BMJ does not warrant the accuracy and reliability of the translations (including but not limited to local regulations, clinical guidelines, terminology, drug names and drug dosages), and is not responsible for any error and/or omissions arising from translation and adaptation or otherwise.

Open access This is an open access article distributed in accordance with the Creative Commons Attribution Non Commercial (CC BY-NC 4.0) license, which permits others to distribute, remix, adapt, build upon this work non-commercially, and license their derivative works on different terms, provided the original work is properly cited, appropriate credit is given, any changes made indicated, and the use is non-commercial. See: http://creativecommons.org/licenses/by-nc/4.0/.

\section{ORCID iDs}

Ellaha Kakar http://orcid.org/0000-0002-7472-308X

M Klimek http://orcid.org/0000-0002-0122-9929

\section{REFERENCES}

1 De Oliveira GS, Holl JL, McCarthy RJ, et al. Overestimation of mortality risk and preoperative anxiety in patients undergoing elective general surgery procedures: a propensity matched analysis. Int $J$ Surg 2014;12:1473-7.

2 Robleda G, Sillero-Sillero A, Puig T, et al. Influence of preoperative emotional state on postoperative pain following orthopedic and trauma surgery. Rev Lat Am Enfermagem 2014;22:785-91.

3 Maranets I, Kain ZN. Preoperative anxiety and intraoperative anesthetic requirements. Anesth Analg 1999;89:1346-51.

4 Kindler $\mathrm{CH}$, Harms C, Amsler F, et al. The visual analog scale allows effective measurement of preoperative anxiety and detection of patients' anesthetic concerns. Anesth Analg 2000;90:706-12.

5 Gras S, Servin F, Bedairia E, et al. The effect of preoperative heart rate and anxiety on the propofol dose required for loss of consciousness. Anesth Analg 2010;110:89-93.

6 Mijderwijk H, van Beek S, Klimek M, et al. Lorazepam does not improve the quality of recovery in day-case surgery patients: a randomised placebo-controlled clinical trial. Eur $J$ Anaesthesiol 2013:30:743-51.

7 Ip HYV, Abrishami A, Peng PWH, et al. Predictors of postoperative pain and analgesic consumption: a qualitative systematic review. Anesthesiology 2009;111:657-77.

8 Sommer M, de Rijke JM, van Kleef M, et al. The prevalence of postoperative pain in a sample of 1490 surgical inpatients. Eur $J$ Anaesthesiol 2008;25:267-74.

9 Cao X, Elvir-Lazo OL, White PF, et al. An update on pain management for elderly patients undergoing ambulatory surgery. Curr Opin Anaesthesiol 2016;29:674-82.
10 Gan TJ. Poorly controlled postoperative pain: prevalence, consequences, and prevention. J Pain Res 2017;10:2287-98.

11 Kumar A, Dubey PK, Ranjan A. Assessment of anxiety in surgical patients: an observational study. Anesth Essays Res 2019;13:503-8.

12 Neuendorf R, Harding A, Stello N, et al. Depression and anxiety in patients with inflammatory bowel disease: a systematic review. J Psychosom Res 2016;87:70-80.

13 Kappelman MD, Long MD, Martin C, et al. Evaluation of the patient-reported outcomes measurement information system in a large cohort of patients with inflammatory bowel diseases. Clin Gastroenterol Hepatol 2014;12:1315-23.

14 Byrne G, Rosenfeld G, Leung Y, et al. Prevalence of anxiety and depression in patients with inflammatory bowel disease. Can J Gastroenterol Hepatol 2017;2017:1-6.

15 Shoar S, Naderan M, Aghajani M, et al. Prevalence and determinants of depression and anxiety symptoms in surgical patients. Oman Med J 2016;31:176-81.

16 Ghoneim MM, O'Hara MW. Depression and postoperative complications: an overview. BMC Surg 2016;16:5.

17 Fu VX, Oomens $\mathrm{P}$, Klimek M, et al. The effect of perioperative music on medication requirement and hospital length of stay: a metaanalysis. Ann Surg 2020;272:961-72.

18 Fu VX, Oomens P, Sneiders D, et al. The effect of perioperative music on the stress response to surgery: a meta-analysis. J Surg Res 2019;244:444-55.

19 Kühlmann AYR, van Rosmalen J, Staals LM, et al. Music interventions in pediatric surgery (the music under surgery in children study). Anesthesia \& Analgesia 2020;130:991-1001.

20 Carroll JK, Cullinan E, Clarke L, et al. The role of anxiolytic premedication in reducing preoperative anxiety. Br J Nurs 2012;21:479-83.

21 Buscemi N, Vandermeer B, Friesen C, et al. The efficacy and safety of drug treatments for chronic insomnia in adults: a meta-analysis of RCTs. J Gen Intern Med 2007;22:1335-50.

22 Holbrook AM, Crowther R, Lotter A, et al. Meta-Analysis of benzodiazepine use in the treatment of insomnia. CMAJ 2000;162:225-33.

23 National Institutes of Health. National Institutes of health state of the science conference statement on manifestations and management of chronic insomnia in adults, June 13-15, 2005. Sleep 2005;28:1049-57.

24 Nowell PD, Mazumdar S, Buysse DJ, et al. Benzodiazepines and zolpidem for chronic insomnia: a meta-analysis of treatment efficacy. JAMA 1997;278:2170-7.

25 Zaal IJ, Devlin JW, Hazelbag M, et al. Benzodiazepine-associated delirium in critically ill adults. Intensive Care Med 2015;41:2130-7.

26 van Beek S, Kroon J, Rijs K, et al. The effect of midazolam as premedication on the quality of postoperative recovery after laparotomy: a randomized clinical trial. Can J Anaesth 2020;67:32-41.

27 Brummett CM, Waljee JF, Goesling J, et al. New persistent opioid use after minor and major surgical procedures in US adults. JAMA Surg 2017;152:e170504

28 Halfens R, Cox K, Kuppen-Van Merwijk A. Effect of the use of sleep medication in Dutch hospitals on the use of sleep medication at home. J Adv Nurs 1994;19:66-70.

29 Carter JE, Pyati S, Kanach FA, et al. Implementation of perioperative music using the consolidated framework for implementation research. Anesth Analg 2018;127:623-31.

30 Hebert CA, Hancock K, McConnell ES. Implementation of individualized music in long-term care: application of the PARiHS framework. J Gerontol Nurs 2018;44:29-38.

31 Sharda N, Mattoon E, Matters L, et al. Bach to the basics: implementation and impact of a postoperative, inpatient personalized music program for older adults. J Perianesth Nurs 2019;34:347-53.

32 Proctor E, Silmere H, Raghavan R, et al. Outcomes for implementation research: conceptual distinctions, measurement challenges, and research agenda. Adm Policy Ment Health 2011;38:65-76.

33 Damschroder LJ, Aron DC, Keith RE, et al. Fostering implementation of health services research findings into practice: a consolidated framework for advancing implementation science. Implement Sci 2009;4:50.

34 Birken SA, Powell BJ, Shea CM, et al. Criteria for selecting implementation science theories and frameworks: results from an international survey. Implement Sci 2017;12:124.

35 Ljungqvist $\mathrm{O}$, Scott M, Fearon KC. Enhanced recovery after surgery: a review. JAMA Surg 2017;152:292-8.

36 Powell BJ, Waltz TJ, Chinman MJ, et al. A refined compilation of implementation strategies: results from the expert recommendations for implementing change (ERIC) project. Implement Sci 2015;10:21. 
37 Proctor EK, Powell BJ, McMillen JC. Implementation strategies: recommendations for specifying and reporting. Implement Sci 2013;8:139.

38 Baker R, Camosso-Stefinovic J, Gillies C, et al. Tailored interventions to overcome identified barriers to change: effects on professional practice and health care outcomes. Cochrane Database Syst Rev 2010;3:CD005470.

39 Pinnock H, Barwick M, Carpenter CR, et al. Standards for reporting implementation studies (STARI): explanation and elaboration document. BMJ Open 2017;7:e013318.

40 Wensing MGR, Grimshaw J. Improving patient care; the implementation of change in clinical practice. 3rd ed. Wiley Blackwell, 2020

41 Richard-Lalonde M, Gélinas C, Boitor M, et al. The effect of music on pain in the adult intensive care unit: a systematic review of randomized controlled trials. J Pain Symptom Manage 2020;59:1304-19.

42 Kelly AM. The minimum clinically significant difference in visual analogue scale pain score does not differ with severity of pain. Emerg Med J 2001;18:205-7.

43 Kamiński JP, Pai A, Ailabouni L, et al. Role of epidural and patientcontrolled analgesia in site-specific laparoscopic colorectal surgery. JSLS 2014;18:e2014.00207.

44 Mouawad NJ, Leichtle SW, Kaoutzanis C, et al. Pain control with continuous infusion preperitoneal wound catheters versus continuous epidural analgesia in colon and rectal surgery: a randomized controlled trial. Am J Surg 2018;215:570-6.

45 Hulscher MEJL, Laurant MGH, Grol RPTM. Process evaluation on quality improvement interventions. Qual Saf Health Care 2003;12:40-6. 